\title{
Bioactivity of pyocyanin of Pseudomonas aeruginosa clinical isolates against a variety of human pathogenic bacteria and fungi species
}

\author{
Zahraa Jaafar Jameel ${ }^{1}$, Anaam Fuad Hussain ${ }^{2}$, \\ Muthana Abdulkhader Al-Mahdawi ${ }^{1}$,
} Nuha F. Abed Alkerim ${ }^{3}$, Eman Sabah abd Alrahman ${ }^{1}$
1 Biology Department. College of Science. Diyala University, Iraq.

2 Biotechnology Department. College of Science. Diyala University, Iraq.

3 Department of chemistry, College of Science,Diyala University, Iraq.

Contact information:

Dr. Zahraa Jaafar Jameel.

झdr.zahraa@sciences.uodiyala.edu.iq
Methods: A total of 23 clinical isolates of $P$. aeruginosa were collected from patients admitted to the General Baqubah Hospital during the period from November 2016 through May 2017. All isolates were cultured on Pseudomonas agar and confirmed by biochemical tests as $P$. aeruginosa. Pyocyanin extraction was done by chloroform method and concentration was determined by multiplying the optical density at $520 \mathrm{~nm}$ by 17.072 expressed as $\mu \mathrm{g} / \mathrm{ml}$. Biological activity of pyocyanin was determined by well diffusion procedure.

Results: According to the source of $P$. aeruginosa, the most tested isolates were from ear infection (30\%) followed by wounds (22\%), burns (17\%), urine (13\%) and both stool and diabetic leg ulcer (9\%). Antimicrobial resistant of $P$. aeruginosa isolates were the following: $19(82.6 \%)$ to piperacillin, followed by $10(43.5 \%)$ to aztreonem, $8(34.8 \%)$ to meropenem, $6(26.1 \%)$ to amikacin, $5(21.7 \%)$ to ciprofloxacin, $2(8.7 \%)$ to cefotaxime.The urine isolates produced the largest amount of pyocyanin $(15.894 \mu \mathrm{g} / \mathrm{ml})$. Pyaocyanin have shown antimicrobial activity against the following bacteria: Shigella spp., Staphylococcus aureus and Staphylococcus epidermedis. Also, pyaocyanin can inhibit the following fungi and yeast: Aspergillus niger, Penicillium 
spp., Rhizopus spp, Trichophyton mentagrophyte, Rhodotorula spp., Alternaria alternate, Trichophyton rubrum and Candida spp.

Conclusions: Antimicrobial activity of pyocyanin was more observed against Gram-positive than Gram-negative bacteria, but mostly similar against all fungi (molds and yeast). Cefotaxime was the most active antimicrobial drug against all $P$. aeruginosa isolates

\section{Keywords}

Pseudomonas Aeruginosa, Pyocyanin, Antimicrobial Activity, Iraq.

\section{Introduction}

Pseudomonas aeruginosa is an opportunistic pathogen of human and can often lead to skin infections, otitis externa, septicemia, necrotizing pneumonia, burns and surgical wounds infections [1, 2].

$P$. aeruginosa is an opportunistic pathogen and it is a frequent cause of nosocomial infections, many of which are responsible for high mortality among hospitalized patients in intensive care units (ICU) and immunocompromised patients even when treated with appropriate antibiotics [1-2].

Pseudomonas aeruginosa produces a variety of cell-associated factors and toxin, some of which are responsible for its invasion and the severity of diseases. These include lipopolysaccharide (LPS), phospholipase, proteases, flagella, pili, exotoxins and pyocyanin [3-4].
Pyocyanin is a blue redox active, secondary metabolite, phenazine derivative that it exhibits a paradoxical pro-oxidant property [4]. A zwitterions that canenterbiological membranes, pyocyanin can directly accept electrons from reducing agents such asNADPH and reduced glutathione (Figure 1) and endure redox cycling using oxygen as an electron acceptor resulting in the generation of the reactive oxygen species (ROS), particularly hydrogen peroxide, with resulting induction of oxidative stress and disturbance of intracellular redox homeostasis [5]. Therefore, the important mechanisms of pyocyanin to prokaryotic and eukaryotic cells are related to inhibition cellular respiration [6]

Pseudomonas spp. are also capable of producing organic volatiles, whose in vitro antifungal nature has been demonstrated against Phytophthora-

Figure 1: Chemical structure of reduced and oxidized forms of pyocyanin and mechanizm [5].
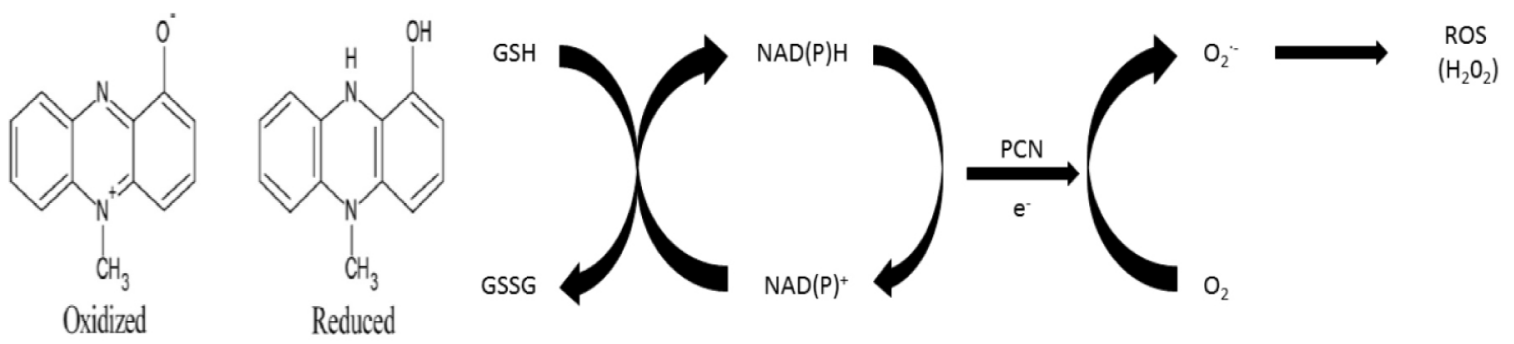
vignae in cowpea [7]. These compounds inhibited sclerotic and ascosporic germination, and mycelial growth of Sclerotinia sclerotiorum, in vitro and in soil tests [8].

This study investigated the bioactivity of pyocyanin produced by fresh $P$. aeruginosa clinical isolates from patients against a variety of bacteria and fungi causing human infections.

\section{Material and Methods}

\section{Bacterial strain isolation and identification}

This study was performed from November,2016 until May, 2017. A total of 23 P. aeruginosa isolates were collected from urine, stool, diabetes leg, ear, burns and wounds infections of patients admitted to General Baqubah Hospital. The isolates were recovered from cultures on Pseudomonas agar, MaCconkey agar and blood agar. All isolates were identified as $P$. aeruginosa using gram-stain, blue-green pigmentation and biochemical characterization [9].

\section{Antibiotic susceptibility testing}

The antimicrobial susceptibility assay was performed on Mueller-Hinton agar using disc-diffusion method, and selection of antibiotics and growth inhibition zones were interpreted according to the Clinical Laboratory Standards Institute [11]. The antimicrobial disks: piperacillin $(100 \mu \mathrm{g})$, cefotaxime $(30 \mu \mathrm{g})$, meropenem $(10 \mu \mathrm{g})$, aztreonem $(30 \mu \mathrm{g})$, amikacin $(30 \mu \mathrm{g})$ and ciprofloxacin $(5 \mu \mathrm{g})$ were obtained from (Mast Group, UK).

\section{Pyocyanin Production}

Nutrient broth was used for the extraction of pigment from each $P$. aeruginosa isolate after incubation for $2-3$ days at $37^{\circ} \mathrm{C}$. The change in color of the pigment to bluish green indicated pigment production [9].

\section{Pyocyanin extraction}

After the color media became bluish, $5 \mathrm{ml}$ of each sample was centrifuged (3000rpm/10min) and 3ml of chloroform added to the supernatant and mixed until blue color is recovered. It was further confirmed by adding $0.2 \mathrm{~N} \mathrm{HCl}$ to the blue color solution which turned to red- pink [12].

\section{Pyocyanin quantitative assay}

The absorbance of each recovered solution was measured at $520 \mathrm{~nm}$. Pyocyanin concentrations were expressed as micrograms of pigment per milliliterof culture and were determined by multiplying theoptical density at $520 \mathrm{~nm}$ by 17.072, according to the absorbance of pyocyanin at $520 \mathrm{~nm}$ in acid solution. [13].

\section{Pyocyanin purification}

$0.4 \mathrm{M}$ borate- $\mathrm{NaOH}$ buffer $(\mathrm{pH}=10)$ was added to the pink solution until the color turned to blue and again extracted by chloroform. This step was repeated 2 to 3 times resulting pyocyanin in chloroform in a clear blue solution. Finally pyocyanin powder was collected by evaporating the chloroform and weighted then rehydration by $1 \mathrm{ml}$ of sterilized distilled water [14].

\section{Biological activity of recovered pyocyanin}

The assay of antibacterial and antifungal of pyocyanin was carried out in well diffusion technique $[15,16]$. Wells with diameter size $6 \mathrm{~mm}$ were made using Mueller-Hinton agar petri dish. Each one isolate of the following pathogenic bacteria were tested: Escherichia coli, Klebsiella pnemoniae, Shigella, Proteus spp., Acinetobacter spp., Staphyllococcus aureus and Staphyllococcus epidermedis. pathogenic fungi and yeast namely Aspergillus niger, Penicillium spp., Rhizopus spp, Trichophyton mentagrophyte, Rhodotorula spp., Alternaria alternate, Trichophyton rubrum and Candida spp. The growth of each isolate was swabbed on the surface of nutrient agar and $100 \mu$ l of pyocyanin solution $(167 \mathrm{mg} / \mathrm{ml})$ were 
added in to the well. Petri dishes were incubated at $37^{\circ} \mathrm{C}$ for $24-48$ hours for bacteria and yeast, while kept for other fungi for up to one month. The inhibition zone in each well was measured by a ruler in $\mathrm{mm}$ unit to determine the biological activity of pyocyanin.

\section{Results}

A total of $23 \mathrm{P}$. aeruginosa isolates were investigated for production of pyocyanin. Distribution of $P$. aeruginosa isolates according to clinical source are shown in Table 1. The majority of isolates were collected from era infections (30\%), followed by wounds $(22 \%)$, burns $(17 \%)$, urine $(13 \%)$ and each leg diabetes and stools (9\%).

\section{Suscptibility to antibiotics}

The results of antimicrobial susceptibility test of 23 $P$. aeruginosa isolates have shown high resistance rate $(82.6 \%)$ to piperacillin, followed by $(43.5 \%)$

Table 1. Distribution of $P$. aeruginosa isolates according to clinical source

\begin{tabular}{|l|c|c|}
\hline \multicolumn{1}{|c|}{ Source } & P. aeruginosa isolates & $\%$ \\
\hline Ear & No (\%) & 30 \\
\hline Wounds & 7 & 22 \\
\hline Burns & 5 & 17 \\
Urine & 4 & 13 \\
Leg Diabetes & 3 & 9 \\
\hline Stool & 2 & 9 \\
\hline Total & 2 & 100 \\
\hline
\end{tabular}

Table 2. Antimicrobial resistance profile of $23 P$. aeruginosa isolates

\begin{tabular}{|l|c|}
\hline \multirow{1}{*}{\multicolumn{1}{c|}{ Antibiotic }} & Resistant \\
\cline { 2 - 2 } & $\%$ \\
\hline Piperacillin & 82.6 \\
\hline Aztreonem & 43.5 \\
\hline Meropenem & 34.8 \\
Amikacin & 26.1 \\
Ciprofloxacin & 21.7 \\
\hline Cefotaxime & 8.7 \\
\hline
\end{tabular}

to aztreonem (34.8\%), to meropenem $(26.1 \%)$ to amikacin, $(21.7 \%)$, to ciprofloxacin and (8.7\%) to cefotaxime, respectively (Table 2).

\section{Pyocyanin extraction (quantitative assay)}

A total of 6 clinical P. aeruginosa isolates were selected to extraction of pyocyanin according to intensity of blue pigmentation on tested agar.

Figure 2, shows the amount of pyocyanin obtained from each $P$. aeruginosa isolate according to their clinical source. The largest amount of pyocyanin $(15.894 \mu \mathrm{g} / \mathrm{ml})$ was recovered from one urine isolate.

Figure 2: Pyocyanin quantity (concentration ( $\mu \mathrm{g} /$ $\mathrm{ml})=$ O.D. $520 \mathrm{~nm} \times 17.072$ ).

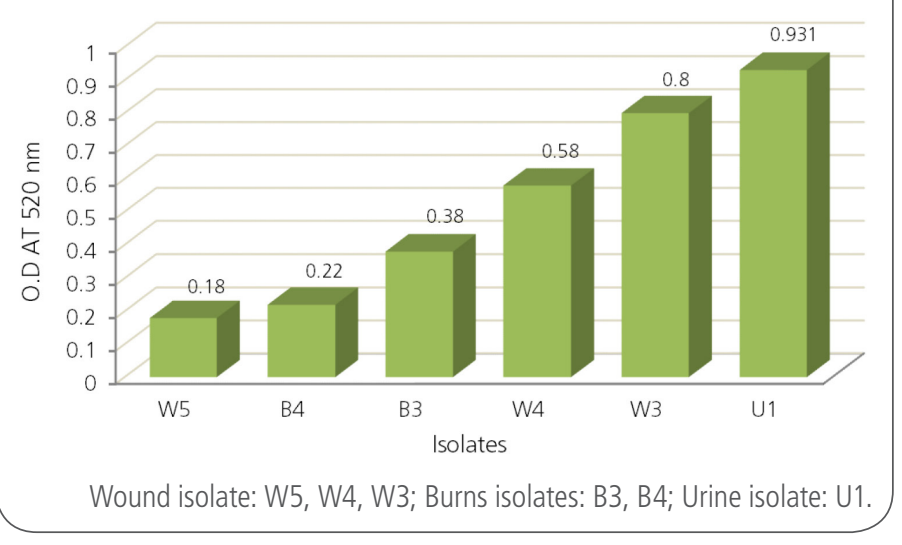

\section{Biological activity of pyocyanin}

The antimicrobial activity of purified pyocyanin (Needle-like crystal) at the concentration $(167 \mathrm{mg} /$ $\mathrm{ml}$ ) was monitored against different microorganisms as shown in Table 3.

Gram-positive such as Staphylococcus epidermidis, Staphylococcus aureus; Gram-negative such as Escherichia coli,proteus spp., Klebsiella spp. and Acinetobacter spp.; molds such as and Yeast as Candid aalbicans. Results shown in table (3) indicate that pyocyanin shows high biological activity against all mentioned organisms, except against Klebsiella pneumonia, Acinetobacter spp., Proteus and Escherichia coli. 
Table 3. Diameter of antimicrobial activity of pyocyanin to various tested microorganisms.

\begin{tabular}{|c|c|c|}
\hline Microorganism & Properties & $\begin{array}{l}\text { Inhibition } \\
\text { zone (mm) }\end{array}$ \\
\hline Shigella spp. & Gram negative & 25 \\
\hline Staphylococcus epidermedis & Gram positive & 23 \\
\hline Staphylococcus aureus & Gram positive & 23 \\
\hline Klebsiella pneumonia & Gram negative & None \\
\hline Acinetobacter spp. & Gram negative & None \\
\hline Proteus spp. & Gram negative & None \\
\hline Escherichia coli & Gram negative & None \\
\hline Penicillium spp. & Mold & $\begin{array}{c}25 \\
\text { (inhibition } \\
\text { of spore } \\
\text { formation)* }\end{array}$ \\
\hline Aspergillusniger & Mold & 25 \\
\hline Aspergillus spp. & Mold & 23 \\
\hline Trichophytonmentagrophyte & Mold & 23 \\
\hline Trichophytonrubrum & Mold & 23 \\
\hline Rhizopus spp.1 & Mold & 23 \\
\hline Alternaria alternate & Mold & 23 \\
\hline Rhodotorula spp. & Mold & 23 \\
\hline Rhizopus spp.2 & Mold & 10 \\
\hline Candida spp.1 & Yeast & 20 \\
\hline Candida spp. 2 & Yeast & 17 \\
\hline
\end{tabular}

\section{Discussion}

Pyocyanin is a blue pigment redox active produced by $P$. aeruginosa, The present study investigated production, purification, characterization and bioactivity of pyocyanin pigments produced by local clinical $P$. aeruginosa isolates. The results have shown that $P$. aeruginosa isolates were most commonly associated with ear infection (30\%) followed by other body sites in our region of Iraq. Many studies carried out in Iraq and other countries demonstrated wide spread of $P$. aeruginosa isolates in clinical specimens. A study of Abbas in Baqubah/Iraq, has found that $P$. aeruginosa first commonly isolated from burns cases (18.18\%), followed by ear (11.6\%), urine $(10.8 \%)$ and wounds (6.25\%) [17]. While the study of Al-Imari in Baghdad/Iraq, has reported the incidence $P$. aeruginosa isolates from certain clinical specimens as follow: urine (22.4\%), ear (18.4\%), sputum (16.3\%), burns (14.3\%) and wounds (12.3\%) [18]. El fouly and coworkers in Cairo, Egypt found that $P$. aeruginosa was the most common cause of urinary tract infection (12.5\%), followed by burns $(10 \%)$ and sputum (9\%) [19]. The reasons of variations in the occurrence rates of $P$. aeruginosa infections may be due to underlining diseases of investigated patients, virulence factors and the local antimicrobial resistance of the organism, and other health conditions [2]. In addition, $P$. aeruginosa is able to act as opportunistic pathogens in human and patients with weakened immune systems, and especially during hospitalization in intensive care units [1-2].

The present study indicated that $P$. aeruginosa isolates have the highst resistant rate to piperacillin $(82.6 \%)$ and less to other antimicrobial agents as shown in Table 2. The study of Al- Alkhozai and Alkabei in Diwanyia, Iraq, reported similar resistance rate in P.aeruginosa isolates to piperacillin (94\%) and high resistance rate to aztreonem (88.5\%) [20]. This study found a resistance rate of $43.5 \%$ to aztreonem, whereas the study in Baqubah/Iraq reported very low resistance rate of 5\% to aztreonem among $P$. aeruginosa isolates [17]. The study of Ameen and coworkers in Pakistan, have found that $P$. aeruginosa resistant to aztreonem was (86.1\%) [21]

It is well established that the development of resistance of $P$. aeruginosa to antibiotics is increasing locally and globally due to the overuse of antibiotics [1]. There are various multiple mechanisms contribute to developing antimicrobial resistance in $P$. aeruginosa, including active efflux, acquisition of various beta-lactamases, decrease outer membrane permeability and target modification [22-23]. Therefore, distinguishing the trends in resistance of $P$. aeruginosa becomes important for choosing the right antibiotic [24].

The production of pyocyanin is exclusive to $P$. aeruginosa, and its production can be improved by 
culture strains on King's A medium which has potassium and magnesium salts in appropriate concentration to defeat fluorescein production (pyoverdine) [2]. Pyocyanin is blue color in alkaline and neutral $\mathrm{pH}$ while red color in acid $\mathrm{pH}$. Any change in the $\mathrm{pH}$ may affect the color of pyocyanin [25]. In addition, the variation in pyocyanin production among different strains could be due to various factors such as lighting conditions. It has been reported that the concentration of pyocyanin was reduced under certain lighting conditions. This decrease was dependent on both the light intensity and wavelength and occurred with light in the ultraviolet and violet region of the spectrum [26-27].

The present study has demonstrated that pyocyanin production by all investigated clinical isolates had antimicrobial activity against Gram-positive Staphyloccocus spp. more than gram negative bacteria and to some extent against yeast such Candida spp. and filamentous fungi. This result is in agreement with those results of Makarand et al. (2007) which have reported that phenazine antibiotic (chemical substance similar to pyocyanin) have antimicrobial activity against strains of Bacillus subtilis and Candida albicans [28].

The study of El fouly in Egypt, found that $P$. aeruginosa isolated from human urine sample produced more pyocyanin concentration than isolates from rice cultivated soil (production titer was 6.3 and 5.9 $\mathrm{mg} / \mathrm{ml}$, respectively) [19], While Ra'oof and Latif, in Iraq, has detected that the $P$. aeruginosa isolate from sputum produced the largest amount of pyocyanin $(12.069 \mu \mathrm{g} / \mathrm{ml})$ [29]. The study of Charyulu et al. (2009), has shown that the secondary metabolite from Pseudomonas which played a significant role in lyses the pathogenic bacterial including MRSA strains [30]. Also, the Sudhakar et al. (2013) showed different antimicrobial activity against E. coli, S. aureus, Proteus sp., and Klebsiella spp. [31], while the study Karpagam et al., (2013) found the pigment produced by $P$. aeruginosa has antifungal activity against Candida sp. and Cryptococcus neoformans [32].
In conclusion, pyocyanin produced by clinical $P$. aeruginosa isolates has various spectrum of antibacterial and antifungal activity against certain Grampositive and Gram-negative bacteria, Candida spp. and filamentous in vitro.

\section{References}

1. Aloush V, Navon-Venezia S, Seigman-Igra Y, Cabili S, Carmeli Y. Multidrug-resistant Pseudomonas aeruginosa: risk factors and clinical impact. Antimicrob Agents Chemother 2006; 50 (1):43-48.

2. Yetkin G, Otlu B, Cicek A, Kuzucu C, Durmaz R. Clinical, microbiologic, and epidemiologic characteristics of Pseudomonas aeruginosa infections in a University Hospital, Malatya, Turkey. Am J Infect Control 2006; 34(4):188-92.

3. Veesenmeyer J, Hauser A, Lisboa T, Rello J. Pseudomonas aeruginosa virulence and therapy: evolving translational strategies. Crit Care Med 2009; 37(5):1777-1786

4. Hall S, McDermott C, Anoopkumar-Dukie S, McFarland A, Forbes A, Perkins AV et al. Cellular Effects of Pyocyanin, a Secreted Virulence Factor of Pseudomonas aeruginosa. Toxins (Basel) 2016; 9; 8(8):1-14

5. Muller M, Merrett N. Pyocyanin Production by Pseudomonas aeruginosa Confers Resistance to Ionic Silver. Antimicrob Agents Chemother. 2014 Sep; 58(9): 5492-5499.

6. Hassan H, Fridovich I. Mechanism of The Antibiotic Action Of Pyocyanine. J Bacteriol 1980; 141(1): 156-163.

7. Fernando W, Linderman R. Inhibition of Phytophthora Vignae and Root Rot of Cowpea by Soil Bacteria. Biological Agriculture and Horticulture 1995; 12(1): 1-14.

8. Fernando W, Ramarathnam R, Krishnamoorthy A, Savchuk S. Identification and Use of Potential Bacterial Organic Antifungal Volatiles in Biocontrol. Soil Biology \& Biochemistry 2005; 37 : 955-964

9. Mahon C, Lehman D, Manuselis G. Textbook of Diagnostic Microbiology, 3rd Edition, Elsevier.2007: P. 508.

10. Clinical and Laboratory Standards Institute (CLSI). Performance standards for antimicrobial susceptibility testing, Seventeenth informational supplement.2012: 27 (1).

11. Frank L, Demoss R. On The Biosynthesis of Pyocyanine1. J Bacteriol 1959; 77(6):776-782

12. Essar D, Eberly L, Hadero A, and Crawfort I. Identification And Characterization Of Genes For Second Anthranilate Synthase In Pseudomonas aeroginosa: Interchaneability of The Two Anthranilate Synthases and Evolutionary Implications. J Bacteriol 1990; $172(2): 884-900$. 
13. Saha, S., Thavas, R., Jayalakshmi, S. Phenazine Pigments From Pseudomonas Aeroginosa And Their Application As Antibacterial Agent And Food Colorants. Res J Microbioy 2008; 3(3):122-128.

14. Chrkraborty P. Textbook Of Clinical Microbiology. 1st Ed. New Central Book Agency.1996:Pp: 381

15. Heatley N. A method for the assay of penicillin. Biochem J 1944; 38

16. Perez M, Bazerque P. Antibiotic assay by agar-well diffusion method, Acta Biol Med Exp 1990; 15: 113.

17. Abbas, Z. Identification And Genetic Study Of Pesudomonus spp Resistant To B- Lactum. M.Sc. Thesis.College of Science, Diyala University, Iraq. 2016:Pp.50-80

18. Al-Imari, H. A Comparative Study On The Antimicrobial And Cytotoxic Effects Of Pyocyanin Produced By Wild Type And Mutant Of Locally Isolated Pseudomonas aeroginosa. M.Sc. Thesis. College of Science, University of Baghdad, Iraq.2011: Pp.67-106

19. El-Fouly A, Sharaf B, Shahin A, Heba A, El-Bialy A, Omara A. Biosynthesis of Pyocyanin Pigment by Pseudomonas aeruginosa. Journal of Radiation Research And Applied Sciences 2015; 8: 36-48.

20. Alkhozai Z, Alkabei M. Isolation and Characterization of New Pseudomonas aeruginosa Phages in Al- Diwanyia City. Journal of al Qadisiyah for pure science 2013; 18(3):10-

21. Ameen N, Memon Z, Shaheen S, Fatima G, Ahmed F. Imipenem Resistant Pseudomonas aeruginosa:The fall of the final quarterback. Pak J Med Sci 2015, 31(3):561-565.

22. Livermore D. Multiple mechanisms of antimicrobial resistance in Pseudomonas aeruginosa: our worst nightmare? Clin Infect Dis 2002; 34: 634-640.

23. Strateva T, Yordanov D. Pseudomonas aeruginosa -a phenomenon of bacterial resistance. J Med Microbiol 2009; 58: 11331148 .

24. Yayan J, Ghebremedhin B, Rasche K. Antibiotic Resistance of Pseudomonas aeruginosa in Pneumonia at a Single University Hospital Center in Germany over a 10-Year Period. PLoS ONE 2015; 10(10).

25. Ohfuji K, Sato N, Hamada-Sato N, Kobayashi T, Imada C, Okuma $\mathrm{H}$, Watanabe E. Construction of a Glucose Sensor Based on a Screen-Printed Electrode and a Novel Mediator Pyocyanin from Pseudomonas aeroginosa. Biosensors and Bioelectronics 2004; 19:1237-1244

26. Propst $C$, Lubi L. Light-Mediated Changes in Pigmentation of $P$. aeruginosa Cultures. J Gen Microbiol 1979; 113:261-266.

27. Whooley M, Meloighlin A. The Regulation of Pyocyanin Production in Pseudomonas aeruginosa. European J App1 Microbiol Biotechnol. 1982; 15:161-166.
28. Makarand R, Prashant D, Bhushan L, Sudhir B. Detection, Isolation and Identification of Phenazine -1-Carboxylic Acid Produced by Biocontrol Strains of Pseudomonas aeruginosa. J Scient Indust Res. 2007; 66:627-631.

29. Ra'oof W, Latif I. In Vitro Study of The Swarming Phenomena and Antimicrobial Activity of Pyocyanin Produced by Pseudomonas aeruginosa Isolated From Different Human Infections. Europ J Scient Res 2010; 47(3):405-421.

30. Charyulu1 M, Sekaran G, Suseela Rajakumar G, Gnanamani A. Antimicrobial activity of secondary metabolite from marine isolate, Pseudomonas sp. against Gram positive and negative bacteria including MRSA. IJEB 2009; 47: 964-968

31. Sudhakar T, Karpagam S, Sabapathy S. Analysis of Pyocyanin Compound and its Antagonistic Activity Against Phytopathogens. Inter J Chem Tech Res 2013; 5(3): 1101-1106

32. Karpagama S, Sudhakar T, Lakshmipathy M. Microbicidal Response of Pyocyanin Produced by $P$. aeruginosa Toward Clinical Isolates of Fungi. JPPS 2013; 5(3): 870-873

\section{Publish in The International Arabic Journal of Antimicrobial Agents}

The Journal is an open access peer-reviewed journal that publishes scientific papers about all aspects of antimicrobials. The journal will publish original research articles, reviews, brief reports and case reports dealing with basic and clinical antibacterial agents, antiviral, antiprotozoals, antituberculuous, antifungal and antihelminthes agents. All manuscripts must be prepared in English, and are subject to a rigorous and fair peer-review process. Accepted papers will immediately appear online. The journal aims to advance the knowledge, attitude and the research of chemotherapy in the Arabic world in cooperation with international, national scientific and public societies as well as research centers with similar aims and objectives. 